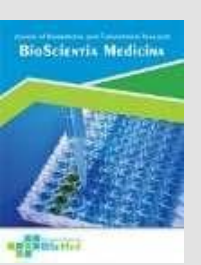

Bioscientia Medicina: Journal of Biomedicine \& Translational Research

Journal Homepage: www.bioscmed.com

\title{
Gitelman Syndrome in a 32-Years-Old Female Patient
}

\section{Andikha Putra ${ }^{*}$, Harnavi Harun ${ }^{1}$}

${ }^{1}$ Department of Internal Medicine, Faculty of Medicine, Universitas Andalas/Dr. M. Djamil General Hospital, Padang, Indonesia

\section{A R T I C L E I N F O}

\section{Keywords:}

Gitelman syndrome

Potassium

Autosomal recessive disorder

Trousseau sign

Hypomagnesemia

\section{*Corresponding author:}

Andikha Putra

\section{E-mail address: andikaputra2146@gmail.com}

All authors have reviewed and approved the final version of the manuscript.

\section{https://doi.org/10.37275/bsm.v6i3.464}

\begin{abstract}
A B S T R A C T
Background. Gitelman syndrome is an autosomal recessive disorder that is milder than Bartter syndrome and is often not diagnosed until late childhood or even adulthood. However, this syndrome is usually symptomatic and can result in serious clinical manifestations, such as muscle spasms that can be severe and involve the hands and feet. These symptoms are frequently observed in almost all patients, partly due to hypokalemia and hypomagnesemia. Case presentation. A 32-year-old female patient is being treated in the Internal Medicine ward of Dr. M. Djamil Padang General Hospital with the main complaint of cramping of the left hand, which has gotten worse since 1 day ago. The patient also complained that both legs were prone to the cramping that went on and on since 1 month ago. The patient had been hospitalized with the same complaint and is currently taking slow-release potassium (KSR) medication. Examination of the patient's limbs revealed a positive trousseau sign and carpopedal spasms. Laboratory examination of the patient showed potassium levels of $1.8 \mathrm{mmol} / \mathrm{L}$, calcium levels of $5.7 \mathrm{mg} / \mathrm{dl}$, Magnesium levels of $0.8 \mathrm{mg} / \mathrm{dl}$, and electrolyte disturbances in the patient's urine. ECG results revealed a prolonged QT interval. Conclusion. Gitelman syndrome is an autosomal recessive disorder and often goes undiagnosed. However, this syndrome is usually symptomatic and can lead to serious clinical manifestations. Most patients require oral potassium and magnesium supplementation, as drug therapy is usually not fully effective.
\end{abstract}

\section{Introduction}

Gitelman syndrome is an autosomal recessive disorder that is milder than Bartter syndrome and is often not diagnosed until late childhood or even adulthood. However, the syndrome is usually symptomatic and can result in serious clinical manifestations, such as cramps that can be severe and involve the hands and feet and are frequently observed in almost all patients, partly due to hypokalemia and hypomagnesemia. Patients may also present with tetany (approximately 10\% of patients) especially if there is an associated disturbance in magnesium absorption (vomitus, diarrhea). Severe weakness was also observed in some patients as well as lower than normal blood pressure, consistent with renal salt leakage. Polyuria and nocturia are found in $50-80 \%$ of patients, can also be accompanied by salt addiction which indicates cause by salt and water loss. Concentration is usually preserved because the function in the ascending medullary limb is relatively intact. Some patients may present with early disease onset accompanied by growth retardation. ${ }^{1-4}$

Patients with Gitelman syndrome have mutations in the gene encoding the thiazide-sensitive $\mathrm{Na}-\mathrm{Cl}$ cotransporter in the distal tubule, often leading to disruption of the cotransporter cellular route (figure 1). Disruption of these transporters can cause both 
magnesium leakage and often decreased calcium excretion, similar to that induced by thiazide therapy and in contrast to the hypercalciuria seen in barter syndrome.

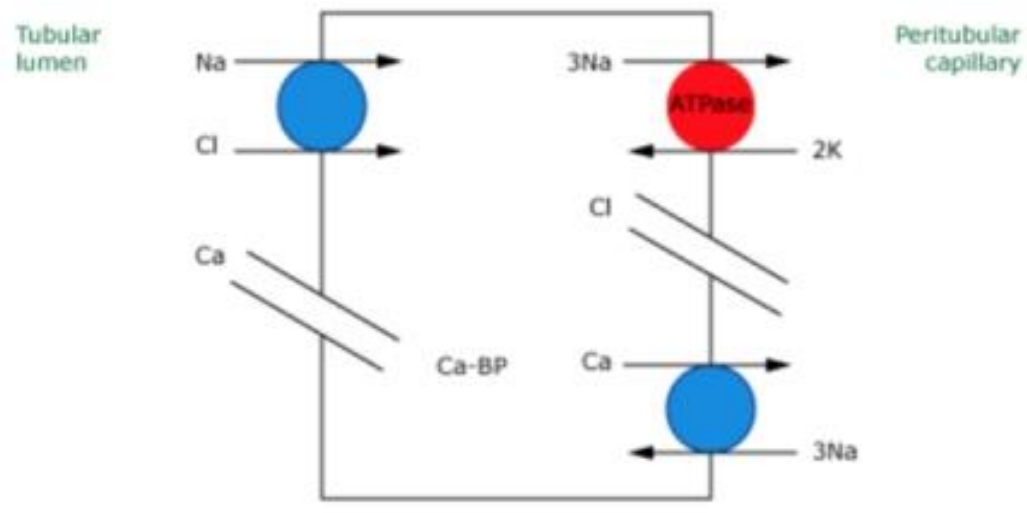

Figure 1. Cotransporter system in the distal tubule; the role of prostaglandins 5

Prostaglandins, which can stimulate renin release directly, contribute to the electrolyte abnormalities in Bartter syndrome, as demonstrated by increased urinary prostaglandin excretion and the ability of prostaglandin synthesis inhibitors to reverse most of the hormonal and biochemical changes, except for primary disturbances of sodium reabsorption. ${ }^{4,5}$

In comparison, prostaglandin excretion appears to be normal and the use of prostaglandin synthesis inhibitors provides little benefit in Gitelman syndrome. These differences are similar to changes in response to diuretic administration. Ansa diuretics (Bartter's syndrome) increase urinary prostaglandin excretion, not least by increasing prostaglandin synthesis in the thick ascending limb. Thiazide diuretics (same as Gitelman syndrome) produce only a small or no increase in prostaglandin secretion. ${ }^{5}$ The urine chloride concentration varies, from high when the diuretic is still active to low when it is no longer working. Patients with Bartter or Gitelman syndrome tend to be euvolemic, with chloride excretion consistent with intake. The net effect of urine chloride concentration is above $40 \mathrm{mEq} / \mathrm{L} .6$

The ability to differentiate the various types of Gitelman from Bartter syndrome can be difficult, because of variations in clinical phenotype and overlapping ages of clinical presentation. Since the genes that cause Gitelman and Bartter syndrome have been identified and described, a genetic diagnosis of both disorders is possible. However, such analyzes are only available in a few research laboratories due to the large size of most of the genes responsible, a large number of recognized mutations and the absence of gene-specific markers, intrafamilial heterogeneity, and high cost. 6

The tubular abnormalities in Gitelman syndrome are irreversible, as a result of which therapy should be lifelong and aim to minimize secondary prostaglandin aldosterone elevations. A combination of nonsteroidal anti-inflammatory drugs (NSAIDs) and potassiumsparing diuretics (spironolactone or amiloride, at a dose of 300-400 mg daily to completely block distal potassium secretion) can increase plasma potassium concentrations toward normal, partially correcting metabolic alkalosis and hypomagnesemia.6,7 A similar improvement in the electrolyte state picture can be achieved with the use of ACE blockers that decrease the production of angiotensin II and aldosterone. However, acute reductions in angiotensin II levels can cause symptomatic hypotension in some cases, this problem is often transient and can be minimized by the use of low initial doses. ${ }^{7}$ Most patients require oral potassium and magnesium supplementation, as drug therapy is usually not fully effective. However, restoration of normal magnesium and potassium levels is often difficult to achieve. Diarrhea often limits the dose of magnesium that can be administered and the magnesium that is absorbed tends to be re-excreted in 
the urine. ${ }^{7}$

\section{Case Presentation}

A 32-years-old female patient was treated in the Internal Medicine ward of Dr. M. Djamil Padang General Hospital with the main complaint of cramping in the left hand which has been getting worse since 1 day ago. In the history of the present illness, it is known that the cramps have been felt since 1 week ago and are felt continuously, enough so that the left hand is difficult to move. The patient also complains that both legs are easy to experience cramps that come and go since 1 month ago, especially after walking a lot. There were no complaints of vomiting, fever, cough, shortness of breath, chest pain, or complaints of urination or defecation. The patient had been hospitalized with the same complaint before being admitted and is currently taking Slow Release Potassium (KSR) medication.

The patient was admitted to the hospital seeming moderately ill with an awareness state of Cooperative Compos Mentis. Examination of vital signs was found to be within normal limits. On physical examination, the patient had a positive Chvostek's sign. Examination of the patient's limbs revealed a positive trousseau sign and carpopedal spasm. In the general status of the patient, examination of the head, eyes, lungs, heart, and abdomen were all within normal limits.

Laboratory examination of the patient showed potassium levels of $1.8 \mathrm{mmol} / \mathrm{L}$, Calcium levels of $5.7 \mathrm{mg} / \mathrm{dl}$, and Magnesium levels of $0.8 \mathrm{mg} / \mathrm{dl}$. ECG results revealed sinus rhythm with a prolonged QT interval. In the patient's urine electrolyte results, urine chloride level was $211 \mathrm{mmol} / \mathrm{L}$ and urine magnesium was $<1.81 \mathrm{mg} / \mathrm{dl}$ with a TTKG value of 17. Routine blood results, peripheral blood images, urinalysis, and routine stools in the patient were found to be within normal limits.

\section{Discussion}

A 32-years-old female patient has been treated in the internal medicine ward of DR. M. Djamil General
Hospital with the final diagnosis of Gitelman syndrome and hypocalcemia. The diagnosis of Gitelman syndrome and hypocalcemia in the patient was established from her medical history, physical examination, and further investigations. In this patient, from the history, she had complaints of cramps in her left hand that had to worsen since 1 day ago. The cramps were felt since 1 week ago. The patient's legs also have been cramping since 1 month ago, especially when she walks a lot. The patient has been treated with the same complaints and is currently taking slowrelease potassium (KSR) drugs. Bordoni in 2018 stated that muscle cramps are continuous involuntary contractions that are painful and localized to a specific muscle group, muscle, or muscle fiber. Several things can cause muscle cramps, namely the use of drugs such as diuretics and calcium channel blockers, dehydration, muscle fatigue after overuse, pregnancy, arterial disease in the legs, and electrolyte disturbances. In the patient, the critical value of calcium levels was $5.7 \mathrm{mg} / \mathrm{dl}$ and potassium 1.8 $\mathrm{mmol} / \mathrm{L}$.

Clinically, the main symptom of hypocalcemia is increased neuromuscular irritability, which can include tingling in the fingertips and around the mouth. Cooper et al in 2008 stated that extracellular calcium concentration is important in maintaining the normal function of nerves and muscles. Carpopedal spasm is characteristic of hypocalcemic conditions which in more severe cases can progress to tetany, convulsions, and arrhythmias. The patient found complaints of cramps in the left hand in the form of carpopedal spasms, which had worsened since 1 day before being admitted to the hospital.

On physical examination, the patient had positive Chvostek's sign and Trousseau's sign. Chvostek's sign is twitching of the area around the mouth when tapped on the facial nerve anterior to the ear. Trousseau's sign is a carpal spasm that occurs when the arm is dammed using a sphygmomanometer cuff at a pressure of 20 $\mathrm{mmHg}$ above systolic pressure for 3 minutes. Classic carpal spasms will include wrist flexion, interphalangeal extension, and adduction of the fingers. Symptoms of more severe hypocalcemia can 
occur spontaneous carpopedal spasm, laryngospasm, or bronchospasm, to generalized seizures. The patient found Chovstek's sign when tapped on the front of the patient's ear. Trousseau's sign was also found by examination using a sphygmomanometer cuff at a systolic blood pressure of $140 \mathrm{mmHg}$ (the patient's blood pressure was 120/80 $\mathrm{mmHg}$ ) for 3 minutes. The patient also found carpopedal spasms in the form of wrist flexion, interphalangeal extension, and adduction of the fingers.

On laboratory examination, potassium levels of 1.8 mmol/L showed hypokalemia. Examination of kidney function found urea levels of $15 \mathrm{mg} / \mathrm{dl}$ and creatinine $0.5 \mathrm{mg} / \mathrm{dl}$, indicating normal kidney function. On examination of urine chloride, the level was 211 $\mathrm{mmol} / \mathrm{L}$, indicating an increase in urine chloride level. Blood gas analysis showed metabolic alkalosis with a $\mathrm{Ph}$ value $=7.559$ and an $\mathrm{HCO} 3-$ value $=14 \mathrm{mmol} / \mathrm{L}$. Urine potassium examination revealed a potassium level of $39.6 \mathrm{mmol} /$ day, urine osmolality 211 $\mathrm{mOsm} / \mathrm{kgH} 2 \mathrm{O}$ at a serum osmolarity of 258 $\mathrm{mOsm} / \mathrm{kgH} 2 \mathrm{O}$, with a TTKG $=17$ which indicates that hypokalemia is due to potassium leakage in the kidneys. On examination of urine calcium, $56 \mathrm{mg} / 24$ hours was found, which means that the patient has decreased urine calcium.

Assessment of potassium excretion in the urine is done by calculating the Trans Tubular Potassium Concentration Gradient (TTKG). TTKG shows an estimate of the potassium level in the tubular fluid precisely at the end of the cortical collecting duct. The normal value of TTKG is $8-9$. If the TTKG value $>11$ indicates an increase in urinary potassium excretion, whereas if the TTKG value is $<11$ it indicates an increase in extrarenal potassium excretion in cases of hypokalemia. Based on the hypokalemic pathway approach with metabolic alkalosis, hypomagnesemia, and hypocalciuria, this patient was diagnosed with Gitelman syndrome.

In asymptomatic patients, a serum calcium level of less than $1.9 \mathrm{mmol} / 1$ is an indication for hospitalization. The patient showed clinical and laboratory improvement after receiving $2000 \mathrm{mg}$ of calcium gluconate in $100 \mathrm{cc}$ of $0.9 \% \mathrm{NaCl}$ taken within
1 hour, followed by $1000 \mathrm{mg}$ of calcium gluconate in $500 \mathrm{cc}$ of $0.9 \% \mathrm{NaCl}$ taken within 12 hours (for 3 days) intravenously. The patient was also given Osteocal $1 \mathrm{x}$ $1000 \mathrm{mg}$ orally.

Treatment of hypokalemia, in general, is the administration of oral or intravenous potassium. Giving potassium is preferred in oral form because it is easier to administer. Administration of 40-60 meq can increase potassium levels by 1-1.5 meq/L, while administration of 135-160 meq can increase potassium levels by 2.5-3.5 meq/L. ${ }^{1}$ Intravenous replacement of potassium in the form of $\mathrm{KCl}$ solution (rapid correction) is given if the hypokalemia is severe or the patient is unable to take potassium by mouth. ${ }^{2}$ These patients were given a KCL correction of 25 meq in 200 cc of $0.9 \%$ $\mathrm{NaCl}$ consumed within 4 hours intravenously with indications of hypokalemia $(1.8 \mathrm{mmol} / \mathrm{L})$, which is an absolute indication for intravenous potassium therapy. The choice of oral substitution therapy in patients with Gitelman syndrome is needed to keep potassium levels in patients stable. This therapy is given for life to avoid repeated attack episodes. The patient was given KSR 2 x $600 \mathrm{mg}$ orally. The patient was also given intravenous magnesium correction $8 \mathrm{~g}$ mgSO4 in $500 \mathrm{cc} \mathrm{NaCl} \mathrm{0.9 \%}$ for the first 24 hours and then $4 \mathrm{~g}$ mgSO4 per day for 3 days on an indication of hypomagnesium (Magnesium $0.8 \mathrm{mg} / \mathrm{dl}$ ). After correcting hypokalemia, hypocalcemia, and hypomagnesemia, hand cramps improved. The patient was then discharged home with clinical and laboratory improvements.

\section{Conclusion}

Gitelman syndrome is an autosomal recessive disorder that often goes undiagnosed. However, this syndrome is usually symptomatic and can lead to serious clinical manifestations. The tubular abnormalities in Gitelman syndrome are irreversible, as a result, therapy should be lifelong and aimed to minimize secondary prostaglandin aldosterone elevations. Most patients require oral potassium and magnesium supplementation, as drug therapy is usually not fully effective. However, restoration of normal magnesium and potassium levels is often difficult to achieve. The combination of a non-steroidal 
anti-inflammatory drug (NSAID) and a potassiumsparing diuretic (spironolactone or amiloride, at a dose of 300-400 mg daily to completely block distal potassium secretion) can increase plasma potassium concentration toward normal, partially correcting metabolic alkalosis and hypomagnesemia.

\section{References}

1. Siregar. P. Disorders of air and electrolyte balance. Textbook of Internal Medicine, Volume II, Edition VI: Publishing Center Department of Internal Medicine FKUI Jakarta, Indonesia. 2017; 2243-59

2. Alwi et al. Management in internal medicine: Clinical Practice Guide. Jakarta: International Publishing. 2019; 389-391.

3. Nathana. Maggie. Hypokalemia-Diagnosis and management. Mirror of the World of Medicine273. 2019; 46(2)

4. Roser M, Eibl M, et al. Big round: Gitelman syndrome. Hypertension 2009; 53; 893-897.

5. Knoers NV, Levtchenko E. Gitelman syndrome. Orphanet Journal of Rare Diseases 2008; 3: 22.

6. Bettinelli A, Tossetto C, et al. Electrocardiogram with prolonged QT in Gitelman syndrome. Kidney International 2008; 62: 580-4.

7. Amirlak I, KP Dawson. Bartter's syndrome: An overview. QJ Med 2006; 93: 207-215. 